[15] J. G. Nash and J. W. Holm-Kennedy, "Effect of electron-electron scattering on hot-electron repopulation in n-Si at $77^{\circ} \mathrm{K}$," Phys. Rev. B, vol. 16, p. 2834, 1977.

[16] J V. Faricelli, "Physics of large-signal response of short-channel MESFET's," M.S. thesis, Cornell Univ., Ithaca, NY, 1980.

[17] K. Blotekjaer, "Transport equations for two-valley semiconductors," IEEE Trans, Electron Devices, vol. ED-17, p. 38, 1970.

[18] M. S. Shur, "Influence of non-uniform field distribution on frequency limits of GaAs field-effect transistors," Electron Lett., vol. 12, p. 615,1976 .

[19] C. Jacoboni et al., "A review of some charge transport properties of silicon," Solid-State Electron., vol. 20, p. 77, 1977.

[20] P. M. Smith, M. Inoue, and J. Frey, "Electron velocity in Si and GaAs at very high electric fields," J. Appl. Phys., vol. 37, p. $797,1980$.

[21] T. J. Maloney, "Non-equilibrium electron transport in compound semiconductors," Ph.D. dissertation, Cornell Univ., Ithaca, NY, 1977.

[22] S. Kratzer, "Computer simulations of electron transport in GaAs," M.S. thesis, Cornell Univ., Ithaca, NY, 1978.
[23] P. N. Swartztrauber and R. Sweet, "Efficient fortran subprograms for the solution of elliptic partial differential equations," $\mathrm{Na}$ tional Center for Atmospheric Res., Tech. Note TN/IA-109, 1975.

[24] R. W. Hockney, "POT4-A fast direct Poisson solver for the rectangle allowing some mixed boundary conditions and internal electrodes," IBM Res. Rep., RC-2870.

[25] D. L. Scharfetter and H. K. Gummel, "Large signal analysis of a silicon Read diode oscillator," IEEE Trans. Electron Devices, vol. ED-16, p. 64, 1969.

[26] M. Reiser, "Large scale numerical simulation in semiconductor device modeling," Computing Methods in Applied Mathematics and Engineering, vol. 1, p. 17.

[27] J. Ruch, "Electron dynamics in short channel field effect transistors," IEEE Trans. Electron Devices, vol. ED-19, p. 652, 1972.

[28] R. C. Eden and B. M. Welch, "GaAs digital integrated circuits for ultra-high speed LSI/VLSI," in VLSI: Fundamentals and A pplica. tions, D. F. Barbe, Ed. Berlin, Germany: Springer-Verlag, 1980.

[29] T. Wada and J. Frey, "Physical basis of short-channel MESFET operation," IEEE J. Solid-State Circuits, vol. SC-14, p. 398, 1979.

\title{
Electrical Properties of Multi $p-n$ Junction Devices
}

\author{
JOSEPH KATZ, SHLOMO MARGALIT, AND AMNON YARIV, FELLOW, IEEE
}

\begin{abstract}
The electrical properties of multi p-n junction devices are analyzed. It is found that this type of device possesses bistable characteristics similar to that of a Shockley diode and thus provides an alternative realization of devices for switching applications. The inherently greater current gains involved in the operations of such a device yield in principle higher breakover voltages and higher holding currents. Furthermore, the incorporation of heterostructures in this device introduces a new degree of freedom in tailoring their switching characteristics. Multi $\mathrm{p}-\mathrm{n}$ heterojunction devices operating as SCR lasers were fabricated, and the experimental results are presented.
\end{abstract}

\section{INTRODUCTION}

S INCE THEIR introduction, the Shockley diode [1] and other related devices have found many applications in switching and regulating circuits [2]. Recently the operation of Shockley diodes which function also as AlGaAs injection lasers has been demonstrated [3]. Operation of both homostructure [4] and heterostructure [5] multi p-n GaAs-GaAlAs

Manuscript teceived November 16, 1981; revised February 3, 1982. This work was supported in part by the Jet Propulsion Laboratory, California Institute of Technology, under NASA Contract NAS7-100, the Office of Naval Research and the National Science Foundation.

J. Katz is with the Jet Propulsion Laboratory, California Institute of Technology, Pasadena, CA 91109.

S. Margalit and A. Yariv are with the Department of Electrical Engineering and Applied Physics, California Institute of Technology, Pasadena, CA 91125. devices as injection lasers has also been reported, but no analysis of the electrical properties of such structures has been published.

This paper analyzes the electrical properties of semiconductor devices consisting of many layers of alternating p-and n-type. Incorporation of heterostructures in these devices makes the design of their characteristics more flexible due to the introduction of the additional degree of freedom of the energy band gap difference. It is found that such devices provide an alternative for realizing bistable switching characteristics. Compared to switching devices fabricated from silicon, GaAs devices are less sensitive to high temperatures because of their larger band gap and are inherently faster because of their shorter carrier lifetime. Since the common-base current gain of the transistors that model these devices (see next section) is distributed among all the regions of the structure, different switching conditions are obtained. Mainly it is found that it takes more gain to perform the switching, which results in an increase in the breakover voltages and in the holding currents.

The outline of this paper is as follows: In Section II, a qualitative analysis of multi $p-n$ devices, based on an extended transistor model, is carried out. The results of this analysis show that such structures have bistable characteristics similar to those of a Shockley diode. Sections III and IV analyze 


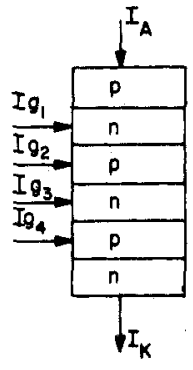

(a)

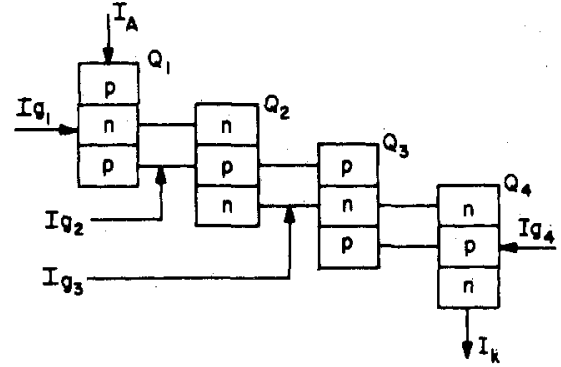

(b)

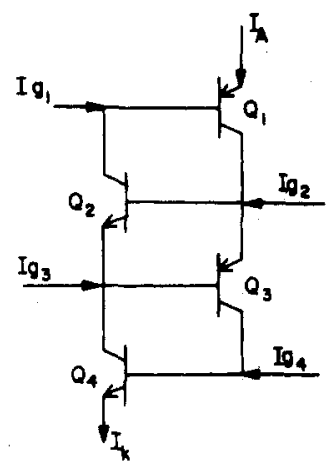

(c)

Fig. 1. Transistor model of a (p-n) $)_{3}$ device. (a) Schematic structure of the device. (b) Decomposition of the device into individual transistors. (c) Equivalent circuit of the device.

quantitatively the device in its two stable states: the forward blocking ("OFF") and the forward conducting ("ON") states, respectively. Finally, Section V describes the fabrication procedure and the experimental results of several types of such devices, and compares the experimental results with the theoretical calculations.

\section{Modified Transistor Model for Multi p-n Structures}

Consider a structure consisting of $2 m$ layers of alternating $\mathrm{p}$ - and n-type, which is denoted by $(\mathrm{p}-\mathrm{n})_{m}$. In this structure the $i$ th junction separates the $i$ th and the $(i+1)$ th layer.

By a direct extension of the two-transistor model for the SCR, one can analyze the structure using a more complicated transistor network. An example of a $(p-n)_{3}$ structure is shown in Fig. 1. Generally, it takes a $2 \times(m-1)$ transistor network to describe a $(\mathrm{p}-\mathrm{n})_{m}$ structure. The $3 \times 2 \times(m-1)$ equations needed to describe the network (three equations for each transistor) are

$$
\begin{aligned}
& I_{B i}+I_{C i}=I_{E i}, \quad i=1,2, \cdots, 2(m-1) \\
& I_{C i}=\alpha_{i} I_{E i}+I_{C O}, \quad i=1,2, \cdots, 2(m-1) \\
& I_{C i}=I_{B, i-1}+I_{E, i-2}+I_{G, i-1} \text {, } \\
& i=2,4,6, \cdots, 2(m-1) \\
& I_{E i}=-I_{B, i-1}+I_{C, i-2},+I_{G, i-1} \text {, } \\
& i=3,5,7, \cdots, 2 m-3 \\
& I_{E \mathbf{1}}=I_{E, 2 m-2}-\sum_{i=1}^{i=2 m-2} I_{G i}
\end{aligned}
$$

where the transistors are assumed to be initially in the cutoff or active region (i.e., the device is in the forward blocking state). $I_{\mathrm{CO} i}$ is the collector to base reverse saturation current of the $i$ th transistor, $\alpha_{i}$ is the common-base current gain of the $i$ th transistor, and $I_{G i}$ is the current generated at the $i$ th gate of the device. The set of equations (1) can be cast in a matrix form

$$
\bar{A} \boldsymbol{I}=\boldsymbol{I}_{\text {drive }} \text {. }
$$

For example, the $(\mathrm{p}-\mathrm{n})_{3}$ structure is described by the follow- ing matrix equation. $\bar{A}$ is given by

$$
\left[\begin{array}{rrrrrrrrrrrr}
1 & 0 & 0 & 0 & 0 & 0 & 0 & 0 & 0 & -1 & 0 & 0 \\
1 & -1 & -1 & 0 & 0 & 0 & 0 & 0 & 0 & 0 & 0 & 0 \\
-\alpha_{1} & 0 & 1 & 0 & 0 & 1 & 0 & 0 & 0 & 0 & 0 & 0 \\
0 & -1 & 0 & 0 & 0 & 1 & 0 & 0 & 0 & 0 & 0 & 0 \\
0 & 0 & 0 & 1 & -1 & -1 & 0 & 0 & 0 & 0 & 0 & 0 \\
0 & 0 & 0 & -\alpha_{2} & 0 & 1 & 0 & 0 & 0 & 0 & 0 & 0 \\
0 & 0 & -1 & 0 & 1 & 0 & 1 & 0 & 1 & 0 & 0 & 0 \\
0 & 0 & 0 & 0 & 0 & 0 & 1 & -1 & -1 & 0 & 0 & 0 \\
0 & 0 & 0 & 0 & 0 & 0 & -\alpha_{3} & 0 & 1 & 0 & 0 & 0 \\
0 & 0 & 0 & -1 & 0 & 0 & 0 & -1 & 0 & 0 & 0 & 1 \\
0 & 0 & 0 & 0 & 0 & 0 & 0 & 0 & 0 & 1 & -1 & -1 \\
0 & 0 & 0 & 0 & 0 & 0 & 0 & 0 & 0 & -\alpha_{4} & 0 & 1
\end{array}\right]
$$

and $\boldsymbol{I}$ and $\boldsymbol{I}_{\text {drive }}$ are given by

$$
I=\left[\begin{array}{c}
I_{E 1} \\
I_{B 1} \\
I_{C 1} \\
I_{E 2} \\
I_{B 2} \\
I_{C 2} \\
I_{E 3} \\
I_{B 3} \\
I_{C 3} \\
I_{E 4} \\
I_{B 4} \\
I_{C 4}
\end{array}\right] I_{\mathrm{drive}}=\left[\begin{array}{c}
-\sum I_{G i} \\
0 \\
I_{C O 1} \\
I_{G 1} \\
0 \\
I_{C O 2} \\
I_{G 2} \\
0 \\
I_{C O 3} \\
I_{G 3} \\
0 \\
I_{C O 4}
\end{array}\right]
$$

Equation (2) can be solved for $I_{A}=I_{E 1}$ with $\left\{\alpha_{i}\right\}$ as a set of parameters. The particular case where $I_{A}$ approaches infinity 


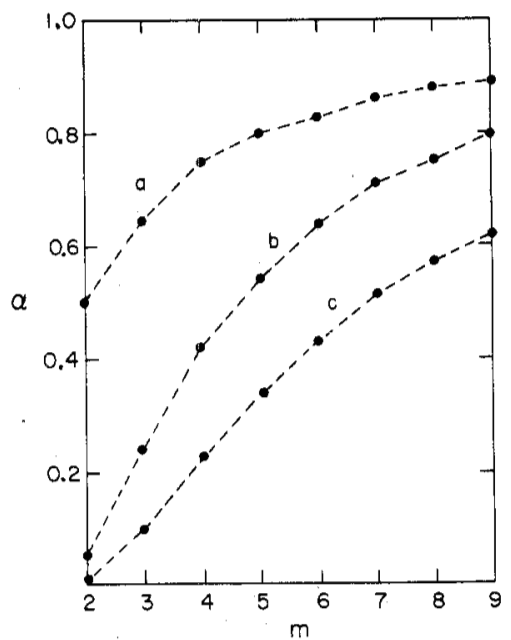

Fig. 2. Common base current gain ( $\alpha$ ) for switching of a (p-n $)_{m}$ device versus $m$. (a) All the transistors are identical $\left(\alpha_{i}=\alpha\right)$. (b) All the odd (or all the even) numbered transistors in the model have $\alpha=0.95$. Shown is $\alpha$ needed from the other transistors for switching. (c) Same as in (b), but with a given $\alpha$ of 0.99 .

(i.e., the determinant of $A$ equals zero) indicates the switching condition. Inspection of the matrix $A$ in (2) shows the following:

1) $(\mathrm{p}-n)_{m}$ structures, with $m \geqslant 2$, cannot possess more than two stable states. This is deduced from the fact that for a given structure, only one set of $\left\{\alpha_{i}\right\}$, at most, yields $I_{A} \rightarrow \infty$.

2) The condition for switching changes, depending on the structure parameters. Some of the results are shown in Fig. 2. In Fig. 2(a) all the transistors are equal; in Fig. 2(b) all the odd (or all the even) numbered transistors in the model have $\alpha=$ 0.95 , and Fig. 2(c) is the same as Fig. 2(b) but with a given $\alpha=0.99$. In Fig. 2(b) and 2(c) shown in the $\alpha$ needed from the other transistors for switching. It is clearly seen that as $m$ increases, the device must have more gain in order to possess two stable states. Structures with insufficient gain remain in the forward blocking state, and when the applied voltage is increased they eventually undergo either avalanche or zener breakdown. One simple explicit expression is obtained for the case where all the transistors have the same gain, i.e., $\alpha_{1}=$ $\alpha_{2}=, \cdots, \triangleq \alpha$. In this case switching occurs in a $(p-n)_{m}$ structure when

$$
\alpha=1-\frac{1}{m} .
$$

For $m=2$ we get the well known result for the SCR $\left(\alpha_{1}=\alpha_{2}=\right.$ 0.5 ).

As a final remark, it is interesting to note that the above analysis can be also carried out for multi p-n structures in which the first layer and the last layer are of the same type (e.g., a p-n-p-n-p structure). In this case it is found that the device behavior does not show bistability, and thus it is similar to that of a transistor. It seems that all generic types of one port low frequency low field semiconductor devices are described by one of the following structures: $p$ (or $n$ ), p-n, p-n-p (or n-p-n) and p-n-p-n.

Fig. 3 helps to explain this fact. In particular, it describes why both $(p-n)_{3}$ and $(p-n)_{2}$ devices have the same basic

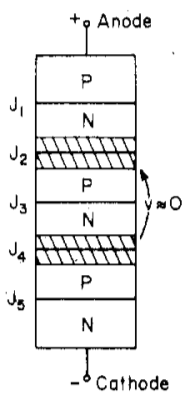

(a)

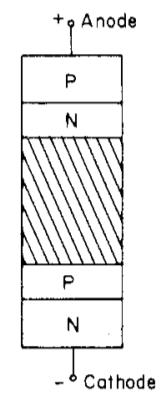

(b)
Fig. 3. Comparison between the generic characteristics of $(p-n)_{3}$ and $(p-n)_{2}$ devices. (a) $(p-n)_{3}$ device. (b) Corresponding $(p-n)_{2}$ device (regular Shockley diode).

electrical characteristics. Fig. 3(a) depicts the device in the forward blocking ("OFF") state. The crosshatched areas represent the depletion regions of the reverse biased junctions $\left(J_{2}\right.$ and $\left.J_{4}\right)$. The junction $J_{3}$ is, in principle, forward biased. However, since the current that flows through the device is very small, there is also a very small voltage drop in the region between $J_{2}$ and $J_{4}$. Since a region with virtually no current and voltage has a little effect on the device, to the external world the device appears basically as if it had the structure depicted in Fig. 3(b), which is a (p-n) $)_{2}$ device. In the forward conducting ("ON") state, all the internal regions in the $(p-n)_{3}$ device are in saturation, which is the same situation as in the $(p-n)_{2}$ device. Of course, the quantitative analysis is different for the two cases, as will be seen in sections which follow.

\section{Solution of Diffusion Equation in the ForWARd Blocking ("OFF") STATE}

In this section the $(p-n)_{m}$ structure in the forward blocking state is analyzed. In this section and in the next one, the indexes on the various parameters refer to either the junctions (e.g., voltages, depletion region recombination currents) or to the layers between the junctions (e.g., diffusion lengths, widths of the layers). The minority-carrier distribution in the forward blocking ("OFF") state is shown in Fig. 4. All the even numbered junctions are reverse biased so that their minority-carrier concentrations are effectively zero. The equation for the current density through the reverse biased junctions is

$$
\begin{aligned}
J=J_{2 i}=M_{2 i}\left[J_{G, 2 i}+J_{p, 2 i+1}(W)\right. & \left.+J_{n, 2 i}(0)\right], \\
& i=1,2, \cdots,(m-1) .
\end{aligned}
$$

$M_{2 i}$ is the avalanche multiplication in the depletion region of the $2 i$ th junction, which, for GaAs, is the same for both electrons and holes and can be approximated by the following empirical formula $[6$, p. 331]:

$$
M_{2 i}\left(V_{2 i}\right)=\left[1-\left(\frac{V_{2 i}}{V_{B D, 2 i}}\right)^{c}\right]^{-1}
$$

where $V_{B D, 2 i}$ is the breakdown voltage of the $2 i$ th $\mathrm{p}-\mathrm{n}$ junction and $c$ is an empirical constant. $J_{G, 2 i}$ is the current density generated in the depletion region of the $2 i$ th junction (e.g., thermal or light generation) which can-to the first orderbe approximated as a constant. $J_{p, 2 i+1}(W)$ is the hole dif- 


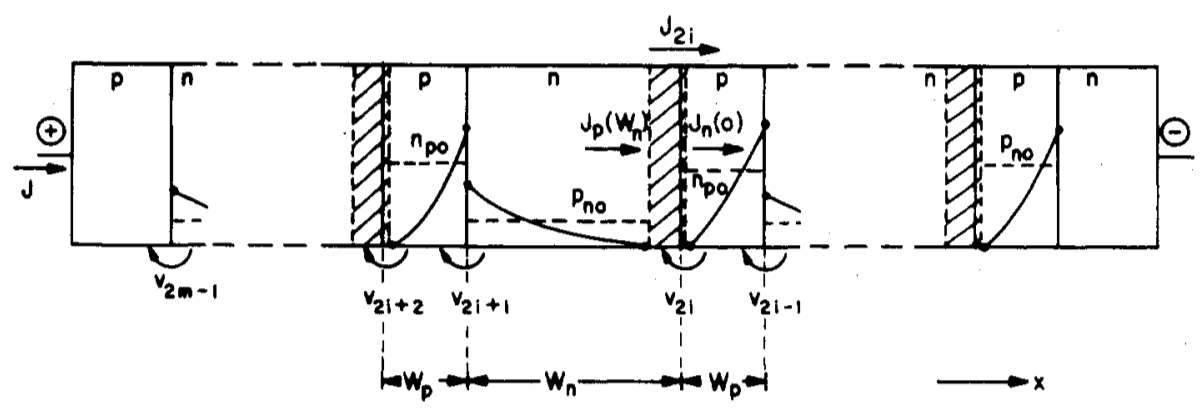

Fig. 4. Minority-carrier distribution across a $(p-n)_{m}$ device in the forward blocking ("OFF") state.

fusion current density entering the junction from the left and is given by

$$
J_{p, 2 i+1}(W)=J_{s p, 2 i+1}\left[\left(e^{\Lambda V_{2 i+1}}-1\right)+\cosh \left(\frac{W_{n, 2 i+1}}{L_{p, 2 i+1}}\right)\right]
$$

where $\Lambda=q / k T$ and $V_{i}$ is the voltage across the $i$ th junction

$$
J_{s p, 2 i+1}=\frac{q D_{p, 2 i+1} p_{n o, 2 i+1}}{L_{p, 2 i+1} \sinh \left(\frac{W_{n, 2 i+1}}{L_{p, 2 i+1}}\right)}
$$

and $J_{n, 2 i}(0)$, the electron diffusion current density entering the junction from the right, is given by

$$
J_{n, 2 i}(0)=J_{s n, 2 i}\left[\left(e^{\Lambda V_{2 i-1}}-1\right)+\cosh \left(\frac{W_{p, 2 i}}{L_{n, 2 i}}\right)\right]
$$

where

$$
J_{s n, 2 i}=\frac{q D_{n, 2 i} n_{p o, 2 i}}{L_{n, 2 i} \sinh \left(\frac{W_{p, 2 i}}{L_{n, 2 i}}\right)}
$$

$n_{p o}, w_{p}, D_{n}, L_{n}$ and $p_{n o}, W_{n}, D_{p}, L_{p}$ are the equilibrium concentration of the minority carriers, the width, the diffusion coefficient and the diffusion length of the minority carriers in the appropriate $p$ and $n$ regions, respectively.

The advantage of using heterostructures in the multi $\mathrm{p}-\mathrm{n}$ devices can be understood from (8) and (10). Since the intrinsic carrier concentration $n_{i}$ in a material is proportional to $\exp (-E g / 2 k T)$ where $E g$ is a band gap, and since, for a given doping level, the minority-carrier concentration (i.e., $n_{p o}$ or $p_{n o}$ ) is proportional to $n_{i}^{2}$, the diffusion currents and hence the current gains $\alpha$ of the transistors that model the device can be modified independently of the doping levels. Thus it is possible to achieve low $\alpha$ transistors in devices with thin layers, i.e., to increase the breakover voltage without sacrificing its temporal response.

The odd numbered junctions are slightly forward biased, so the effect of the depletion region recombination currents must be included. These currents can be approximated by [6, pp. 102-104]

$$
J_{R}=J_{R O} e^{\Lambda V / N_{E}}
$$

where $J_{R O}$ and $N_{E}$ are empirical constarits. In the following calculations it will be assumed that $N_{E}=2$, which is a good approximation for practical devices [6, p. 102-104] .

Since the current is the same throughout the device, the current densities through the even and odd numbered junctions can be equated, so the current density is given by

$$
\begin{aligned}
J= & J_{2 i+1}=J_{p, 2 i+1}(0)+J_{n, 2 i+2}(W)+J_{R, 2 i+1} \\
= & J_{s p, 2 i+1}\left[\left(e^{\Lambda V_{2 i+1}}-1\right) \cosh \left(\frac{W_{n, 2 i+1}}{L_{p, 2 i+1}}\right)+1\right] \\
& +J_{s n, 2 i+2}\left[\left(e^{\Lambda V_{2 i+1}}-1\right) \cosh \left(\frac{W_{p, 2 i+2}}{L_{n, 2 i+2}}\right)+1\right] \\
& +J_{R O, 2 i+1} e^{\Lambda V_{2 i+1} / 2}
\end{aligned}
$$

or

$$
\begin{array}{r}
J=J_{s, 2 i+1}^{*}\left(e^{\Lambda V_{2 i+1}}-1\right)+J_{s, 2 i+1}+J_{R O, 2 i+1} e^{\Lambda V_{2 i+1} / 2}, \\
i=0,1,2, \cdots,(m-1)
\end{array}
$$

where $J_{p, 2 i+1}(0)$ is the hole diffusion current density entering the junction from the right, $J_{n, 2 i+2}(W)$ is the electron diffusion current density entering the junction from the left, $J_{s p}$ and $J_{s n}$ are as defined before in (8) and (10)

$$
\begin{aligned}
& J_{s, 2 i+1} \triangleq J_{s p, 2 i+1}+J_{s n, 2 i+2} \\
& J_{s, 2 i+1}^{*} \triangleq J_{s p, 2 i+1} \cosh \left(\frac{W_{n, 2 i+1}}{L_{p, 2 i+1}}\right)+J_{s n, 2 i+2} \cosh \left(\frac{W_{p, 2 i+2}}{L_{n, 2 i+2}}\right)
\end{aligned}
$$

and $J_{R O, 2 i+1}$ is the recombination current constant of the $(2 i+1)$ th junction.

Note that for $i=0, J_{p}(0)=0$, and for $i=m-1, J_{n}\left(W_{p}\right)=0$, since it can be assumed that the diffusion currents in the two extreme layers are negligible, e.g., these layers are AlGaAs layers with high $\mathrm{Al}$ contents, and thus their values of $n_{p o}$ and $p_{n o}$ are much lower than those of GaAs, because of the differences in the band gap energies.

Using (5) to (13), a closed form expression for the $J$ - $V$ curve of the device can be obtained in the following way. For a given value of $J,(13)$ is a quadratic in

$$
e^{\Lambda V_{i} / 2}, \quad i=1,3,5, \cdots,(m-1) .
$$




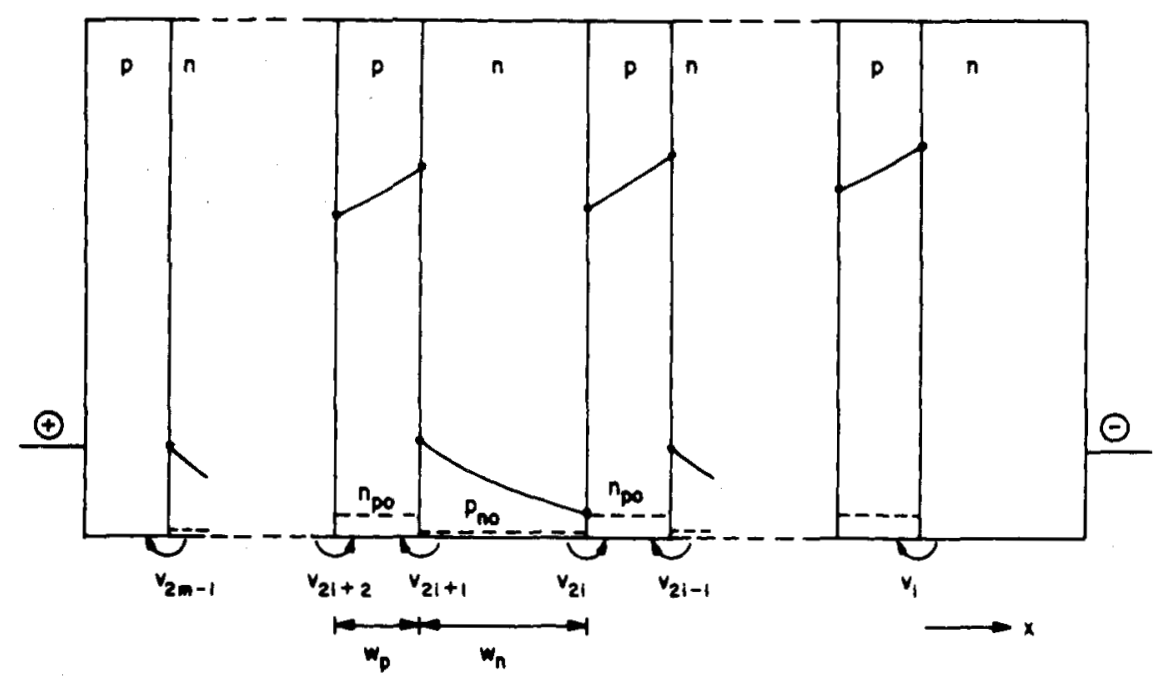

Fig. 5. Minority-carrier distribution across a $(p-n)_{m}$ device in the forward conducting ("ON") state.

Once a solution is obtained for all the odd numbered junctions, (6) can be solved for

$$
V_{i}, \quad i=2,4, \cdots,(m-2)
$$

using (5) and (7) to (10). The sum of all of the junction voltages thus obtained is the total voltage $V$ across the device, corresponding to the assumed value of $J$. The particular form of the resulting expressions is quite complicated, but the calculations are straightforward, as described above. The important parameter $J-V$ curve in the "OFF" state is the breakover voltage $\left(V_{B O}\right)$, which is defined in the same way as for the Shockley diode. At this point $d V / d J=0$. As the current is further increased, the voltage across the device decreases. This is a negative resistance $[d V / d J<0]$ region and thus unstable, leading to the "ON" state. In this new situation the assumption about the junction voltages are no longer valid, and new calculations need to be done.

The breakover voltage of the $(p-n)_{m}$ device is the same as the voltage across $(m-1)$ Shockley diodes operating in series. This is because the structure consists of distinct sections, each isolated between two reverse biased junctions at which the carrier concentration is virtually zero (see Fig. 4). Thus is seen that by increasing $m$ devices can be found with higher breakover voltages.

\section{Solution of the Diffusion Equation in the Forward Conducting ("ON") STAte}

In the forward conducting state all the junctions are forward biased

$$
\left|V_{i}\right| \gg k T / q, \quad i=1,2, \cdots,(2 m-1)
$$

i.e., all the transistors that model the device are in saturation. This is similar to the behavior of the common Shockley diode in the "ON" state. The distribution of the minority-carrier concentration in this state is shown in Fig. 5. (Note the change of sign in the notation for the even numbered junction voltages; now all the junctions are forward biased). The resulting diffusion equations of the entire structure can be written in the form

$$
\bar{B} \boldsymbol{u}+\bar{C} \boldsymbol{w}=J
$$

where

$$
\begin{aligned}
& u=\left|\begin{array}{c}
e^{\Lambda V_{1}}-1 \\
e^{\Lambda V_{2}}-1 \\
\vdots \\
e^{\Lambda V_{2} m-1}-1
\end{array}\right| \\
& w=\left|\begin{array}{c}
e^{\Lambda V_{1} / 2}-1 \\
e^{\Lambda V_{2} / 2}-1 \\
\vdots \\
e^{\Lambda V_{2 m-1 / 2}}-1
\end{array}\right|
\end{aligned}
$$

and

$$
J=\left|\begin{array}{r}
1 \\
-1 \\
\vdots \\
1
\end{array}\right|
$$

The matrix $\bar{B}$, given by

$$
\overrightarrow{\boldsymbol{B}}=\left|\begin{array}{cccccc}
J_{s n 1}^{*} & -J_{s n 2} & 0 & \vdots & & 0 \\
-J_{s n 1} & J_{s n 2}^{*} & -J_{s p 3} & \dot{0} & : & \vdots \\
0 & -J_{s p 2} & J_{s 3}^{*} & -J_{s n 4} & \vdots & \vdots \\
\vdots & \vdots & \vdots & \vdots & & \\
0 & & & & -J_{s p, 2 m-2} & J_{s p, 2 m-1}^{*}
\end{array}\right|
$$


TABLE I

Parameters of a $(p-n) ;$ Structure

\begin{tabular}{|c|c|c|c|c|}
\hline $\begin{array}{l}\text { Layer } \\
\text { Number }\end{array}$ & Type & $\begin{array}{c}\text { A1 } \\
\text { Contents } \\
(x)\end{array}$ & $\begin{array}{c}\text { Doping } \\
\text { Concentration } \\
\times 10^{18}\left[\mathrm{~cm}^{-3}\right]\end{array}$ & $\begin{array}{l}\text { Wideh } \\
{[\mu \mathrm{m}]}\end{array}$ \\
\hline 1 & $n$ & 0.4 & 0.1 & 1.5 \\
\hline 2 & p & 0.0 & 3 & 0.2 \\
\hline 3 & $\mathrm{n}$ & 0.1 & 0.1 & 0.8 \\
\hline 4 & $p$ & 0.0 & 3 & 0.2 \\
\hline 5 & $\mathrm{n}$ & 0.1 & 0.1 & 0.8 \\
\hline 6 & p & 0.0 & 3 & 0.2 \\
\hline 7 & $\mathrm{n}$ & 0.1 & 0.1 & 0.8 \\
\hline 8 & p & 0.0 & 3 & 0.2 \\
\hline 9 & $\mathrm{n}$ & 0.1 & 0.1 & 0.8 \\
\hline 10 & p & 0.0 & 3 & 0.2 \\
\hline 11 & $n$ & 0.1 & 0.1 & 0.8 \\
\hline 12 & p & 0.0 & 3 & 0.2 \\
\hline 13 & n & 0.1 & 0.1 & 0.8 \\
\hline 14 & $\mathrm{P}$ & 0.4 & 1 & 1.5 \\
\hline 15 & $\mathrm{p}^{+}$ & 0.0 & $\approx 10$ & 1.0 \\
\hline
\end{tabular}

TABLE II

Parameters of a $(p-n)_{5}$ Structure

\begin{tabular}{ccccc}
\hline $\begin{array}{l}\text { Layer } \\
\text { Number }\end{array}$ & Type & $\begin{array}{c}\text { Ai } \\
\text { Contents } \\
(\mathrm{x})\end{array}$ & $\begin{array}{c}\text { Doping } \\
\text { Concentration } \\
\times 10^{18}\left[\mathrm{~cm}^{-3}\right]\end{array}$ & $\begin{array}{c}\text { Width } \\
{[\text { Hm] }}\end{array}$ \\
\hline 1 & $\mathrm{n}$ & 0.4 & 0.1 & 1.5 \\
2 & $\mathrm{p}$ & 0.0 & 3 & 0.25 \\
3 & $\mathrm{n}$ & 0.1 & 0.1 & 0.5 \\
4 & $\mathrm{p}$ & 0.1 & 0.1 & 0.5 \\
5 & $\mathrm{n}$ & 0.0 & 3 & 0.25 \\
6 & $\mathrm{p}$ & 0.1 & 0.1 & 0.25 \\
7 & $\mathrm{n}$ & 0.0 & 3 & 1.0 \\
8 & $\mathrm{p}$ & 0.1 & 3 & 0.25 \\
9 & $\mathrm{n}$ & 0.0 & 1 & 1.5 \\
10 & $\mathrm{p}$ & 0.4 & $\approx 10$ & 1.0 \\
11 & $\mathrm{p}+$ & 0.0 & & \\
\hline
\end{tabular}

contains the diffusion contribution to the total current and

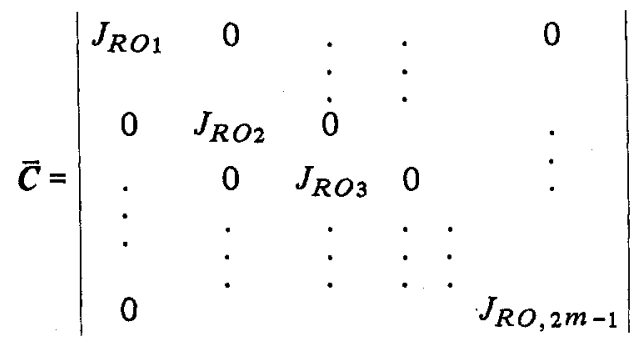

contains the depletion region recombination contribution to to total current. $J_{s p, i}, J_{s n, i}, J_{s, i}^{*}$ and $J_{R O i}$ are given in (8), (10), (15) and (11), respectively. Also

$$
J_{s p, i}^{*}=J_{s p, i} \cosh \left(W_{n, i} / L_{p, i}\right)
$$

and

$$
J_{s n, i}^{*}=J_{s n, i} \cosh \left(W_{p, i} / L_{n, i}\right) .
$$

The derivation of (16) to (21) is based on solving the diffusion equation in each region separately and arranging the individual solutions with the appropriate indexing.

Several calculated results for the devices described in Tables I and II are shown in Figs. 6 and 7, respectively. Part (a) of these figures shows the distribution of the excess minority carriers across the devices. The distribution across the $(p-n)_{5}$ device is much more balanced than in the $(p-n)_{7}$ device. This fact is also clearly demonstrated in part (b) of the figures, which shows the distribution of the recombination current

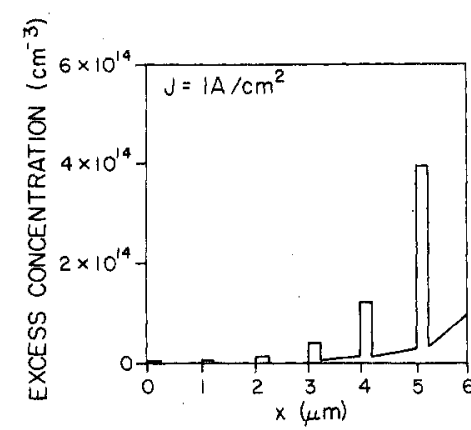

(a)

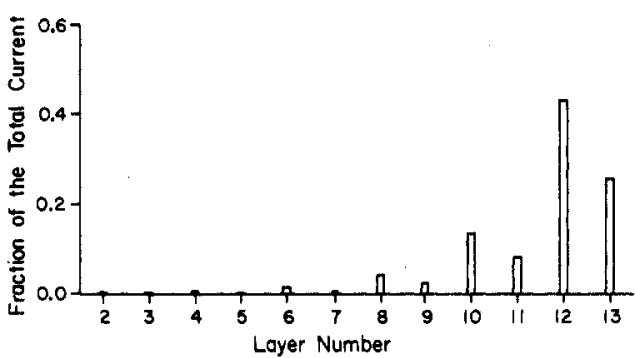

(b)

Fig. 6. "ON" state characteristics of the (p-n $)_{7}$ device described in Table I. (a) Excess minority carriers. (b) Recombination current distribution in the various regions.

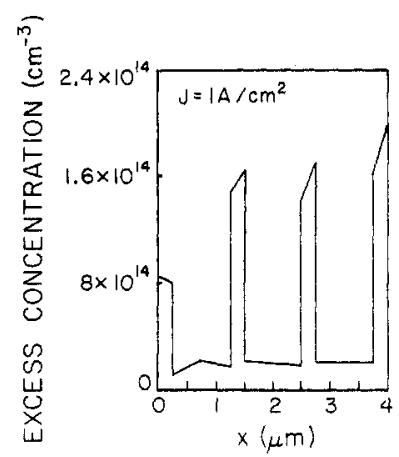

(a)

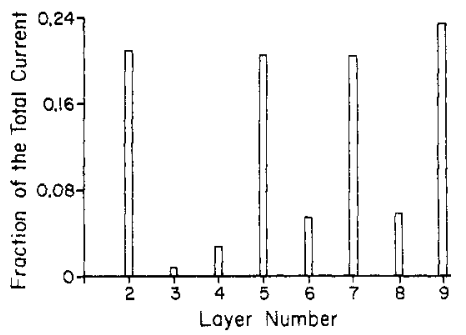

(b)

Fig. 7. "ON" state characteristics of the $(p-n)_{5}$ device described in Table II. (a) Excess minority carriers. (b) Recombination current distribution in the various regions.

across the device. As will be discussed in Section V, it is sometimes desirable to have as uniform distribution in the GaAs regions when possible. When designing a structure for a particular carrier profile, the parameters at our disposal are the number of the layers, their types and widths, and the doping concentration. All these parameters appear in the solution of 


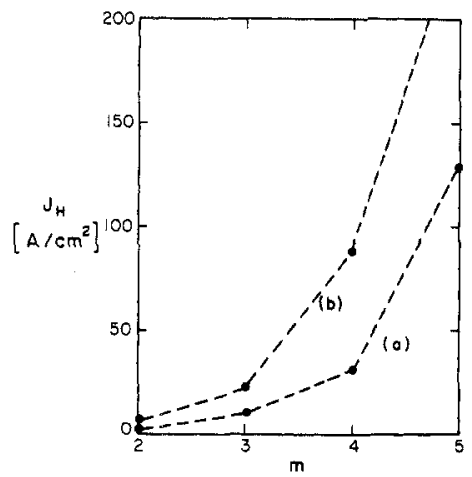

Fig. 8. Calculated dependence of the holding current density $\left(J_{H}\right)$ on the number of layers in the device. (a) $W_{n}=W_{p}=0.5 \mu \mathrm{m}$. (b) $w_{n}=w_{p}=1 \mu \mathrm{m}$.

the diffusion equation, and thus can affect the performance of the device.

The holding current of the device (i.e., the minimum forward current which is required to sustain the "ON" state) can be estimated in the following way. We know that if the current is reduced below the holding value, the device exhibits negative resistance and is unstable. This leads to the "OFF" state described in the preceding section, so the value of $J$ for which $d V / d J=0$ is the holding current density $\left(J_{H}\right)$. This parameter can be found by solving (16) numerically. Calculated dependence of $J_{H}$ on several parameters is shown in Fig. 8. In Fig. 8(a) the width of all the layers is $0.5 \mu \mathrm{m}$, and in Fig. 8(b) the width of all the layers is $1 \mu \mathrm{m}$. The device consists of $(m-1)$ p-n sections of GaAs sandwiched between two layers of high $\mathrm{Al}$ contents AlGaAs. As expected, the holding current density increases with increasing the number of the p-n sections of the device, with increasing the widths of the layers and with increasing the doping levels. The basic cause for this increase is the need to replenish recombined carriers in more and more regions while still maintaining all the layers in saturation. Because of the basic exponential dependence between the current and the voltage in $\mathrm{p}-\mathrm{n}$ devices, the increase in the holding current with increasing the number of sections in the device is larger than the corresponding increase in the breakover voltages.

It should be noted that the above analysis can be easily extended to any arbitrary structure, not necessarily one which consists of alternating $p$ and $n$ regions (e.g., p-p-n-n ...).

\section{Fabrication Procedure and Experimental RESULTS}

The layered structures of $\mathrm{AlGaAs}$ described in this paper were grown by liquid phase epitaxy at $800^{\circ} \mathrm{C}$. Parameters of two typical device structures (e.g., layers types, widths, doping) are described in Tables I and II. Since the number of solution chambers in the graphite boat is smaller than the required number of layers in the structure, the periodic parts of the structure were grown by moving the slide-bar of the boat in both directions between the solutions. In that case, two "dummy" wafers were used, one on each side of the ac-

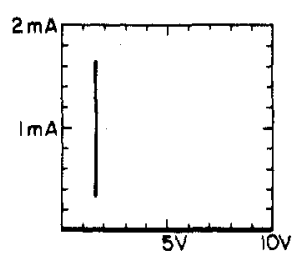

(a)

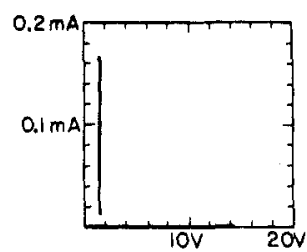

(b)

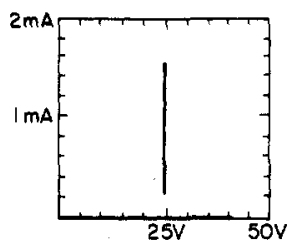

(c)

Fig. 9. Dependence of the $I-V$ curve of $(p-n)_{7}$ devices on the Al contents $(x)$ in the waveguide layers. (a) $x=0.1$. (b) $x=0.2$. (c) $x=0.4$ (no bistability).

tual growth wafer. The dopants used were Ge (p-type), Sn (n-type, for regions with $N_{D} \lesssim 1 \times 10^{18} \mathrm{~cm}^{-3}$ ) and Te (n-type, for regions with $N_{D} \gtrsim 1 \times 10^{18} \mathrm{~cm}^{-3}$ ).

Devices tested only for electrical parameters were etched down to a $100 \times 100 \mu \mathrm{m}^{2}$ mesas, while devices which operated also as injection lasers were etched down to a $100 \mu \mathrm{m}$ mesa in one direction and cleaved to $\approx 300 \mu \mathrm{m}$ length in the perpendicular direction.

$\mathrm{Cr}$-Au was used on the p-type contact and $\mathrm{AuGe} / \mathrm{Au}$ (with a post-deposition alloying at $360^{\circ} \mathrm{C}$ ) was used for the n-type contact.

The first types of devices tested were $(p-n)_{7}$ structures whose order and type of layers, dimensions and doping (but not the $\mathrm{Al}$ contents $(x)$ ) are described in Table I. In particular, the dependence of the $I-V$ curve of the structure on the $\mathrm{Al}$ contents $(x)$ in the layers between the $\mathrm{GaAs}$ regions were investigated. Curves of devices with $x=0.1,0.2$, and 0.4 are shown in Fig. 9. When the Al contents is too high, the current gain of the p-n-p transistors in the device model (these are the transistors which have the $\mathrm{n}$-AlGaAs layers as their base regions) becomes too small to maintain the device in the "ON" state. For $x=0.4$ even reduction in the number of layers, e.g., $(\mathrm{p}-\mathrm{n})_{4}$ is not enough. In this case the obtained $I-V$ curve is that of a transistor in avalanche. The calculated carriers distribution in the device is shown in Fig. 6(a) for $x=0.1$. The carrier concentration is found to be highest in the upper GaAs region, with fewer carriers in the subsequent regions. This result was qualitatively verified in the following way. Instead of etching the devices into the $100 \times 100 \mu \mathrm{m}^{2}$ mesas, they were etched only in one direction and cleaved in the 


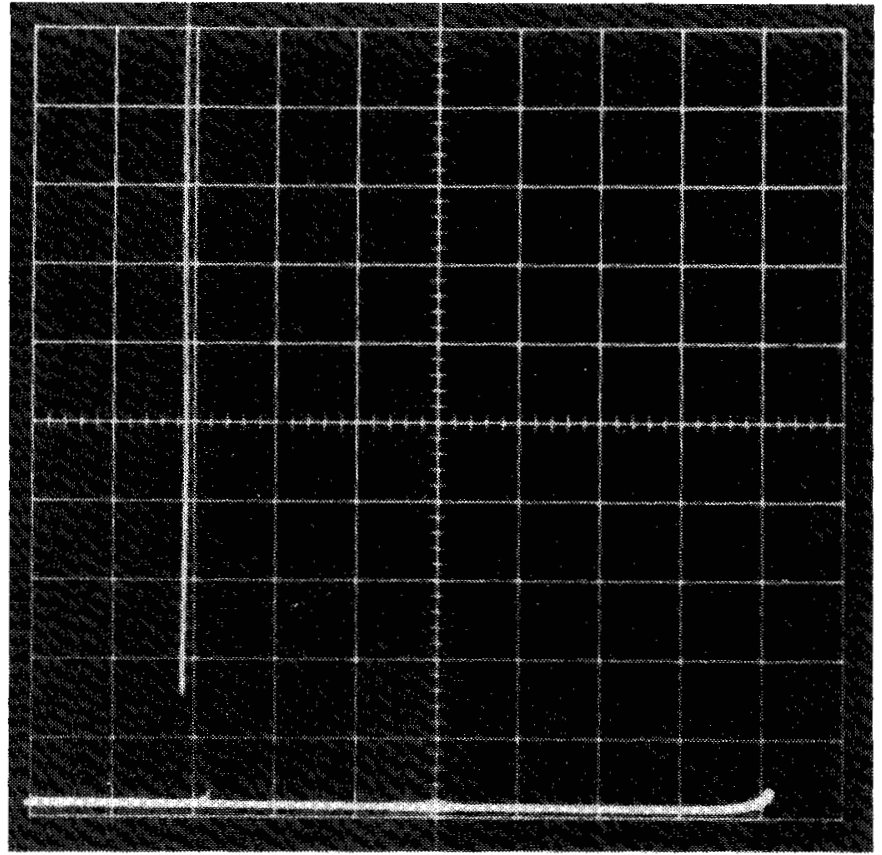

Fig. 10. $I-V$ curve of a $(\mathrm{p}-\mathrm{n})_{5}$ device (horizontal scale: $1 \mathrm{~V} /$ div; vertical scale: $0.1 \mathrm{~mA} / \mathrm{div}$ ).

other dimension (with lengths about $300 \mu \mathrm{m}$ ) thus forming the common Fabri-Perot cavity of semiconductor injection lasers. Below the lasing threshold current of the device, the distribution of the amount of light emitted from each GaAs ("active") region via the spontaneous emission, which is proportional to the carrier concentration in it, was observed to be in agreement with Fig. 6(b). As the current was further increased, it was found that at, or slightly above the threshold $\left(J_{\text {th }} \approx 5.5 \mathrm{kA}-\mathrm{cm}^{-2}\right)$ only the upper active region is lasing, while all the other active regions emitted only spontaneous emission. Only when the current was raised to about $1.4 \mathrm{X}$ $I_{\text {th }}$ did the next active region lase.

Devices of a second type were grown with the goal of equalizing the carrier distribution in all the GaAs layers of the device so that the light emitted will be more evenly distributed. The parameters of the resulting $(\mathrm{p}-\mathrm{n})_{5}$ structure are described in Table II, and its typical $I-V$ curve is shown in Fig. 10. The calculated results on this structure are given in Fig. 6. From Fig. 6(b) it is seen that most of the carriers $(\approx 82$ percent) recombine in the active regions, and the level of recombination currents in the different active regions is uniform to within 10 percent. The breakover voltage $\left(V_{B O}\right)$ of the device is $9 \mathrm{~V}$. Devices with breakover voltages of more than $35 \mathrm{~V}$ were also observed. The value of $V_{B O}$ in each particular device also depends on the amount of leaking due to imperfections. The holding current density $\left(J_{H}\right)$ is about $1.5 \mathrm{~A} \mathrm{~cm}^{-2}$. Lasers made of the $(\mathrm{p}-\mathrm{n})_{\mathbf{5}}$ devices had threshold current density of about $13 \mathrm{kA} \mathrm{cm}^{-2}$, which is comparable to conventional large optical cavity lasers of the same dimensions. More details on the optical characteristics of multi p-n structures can be found in [5], [7].

\section{Conclusions}

The electrical properties of multi p-n junction devices (both homostructures and heterostructures) were analyzed. By using a modified transistor model it was found that devices of this type possess bistable characteristics similar to that of a Shockley diode, and thus they are potentially suitable for switching applications. Among these applications are semiconductor controlled rectifiers with higher breakover voltages and potentially shorter switching times, semiconductor laser devices which also have intrinsic electrical switching capabilities, and large optical cavity lasers with multiple active regions. Quantitative analysis indicated that the inherently greater current gains involved in the operation of such a device yield higher breakover voltages and higher holding currents. Experimental results verified the basic generic characteristics and showed a good fit with the calculated performance.

\section{REFERENCES}

[1] J. L. Moll et al., "p-n-p-n transistor switches," Proc. IRE, vol. 44, pp. 1174-1182, 1956.

[2] F. E. Gentry et al., Semiconductor Controlled Rectifiers. Englewood Cliffs, NJ: Prentice-Hall, 1964.

[3] C. P. Lee et al., "Barrier-controlled low-threshold p-n-p-n GaAs heterostructure laser," Appl. Phys. Lett., vol. 30, pp. 535-538, 1977.

[4] W. F. Kosonocky, R. H. Cornely, and I. J. Hegyi, "Multilayer GaAs injection laser," IEEE $J$. Quantum Electron., vol. QE-4, pp. 175-179, 1968.

[5] J. Katz et al.," "Large optical cavity A1GaAs lasers. with multiple active regions," J. A ppl. Phys., vol. 51, pp. 4038-4041, 1980.

[6] S. M. Sze, Physics of Semiconductor Devices. New York: WileyInterscience, 1969, pp. 102-104, 331.

[7] P. Yeh, A Yariv, and C. S. Hong, "Electromagnetic propagation in periodic stratified media. I. General theory," J. Opt. Soc. Amer., vol. 67, pp. 428-438, 1977. 